\title{
Role of Biomarkers in Cancer Drug Development: A Mini Review
}

\author{
Article by Sayanth Raj ${ }^{1}$, Imran Shareef Syed ${ }^{1}$, Niharika Anand ${ }^{1}$, Jessica Dasari ${ }^{1}$, Rozmina \\ Banu $^{1}$ \\ ${ }^{1}$ MD, Texila American University - College of Medicine, Guyana, South America
}

\begin{abstract}
Biomarkers, or biological markers, are defined in various ways. Some define it as a biologic feature that can be used to measure the presence or progress of disease or the effects of treatment. One goal of biomarker usage in clinical research is to expedite the drug development process to produce drug therapies as efficiently as possible, while maintaining the safety profile. Biomarkers have been used for decades, from monitoring blood pressure to lipid levels. Tumor biomarkers represent an effective tool for tumor diagnosis, treatment, prognosis, and therapeutic monitoring. Ideally, the use of biomarkers in early trials would help predict the likelihood of success or failure of a drug in efficacy trials. Biomarkers can predict drug efficacy more quickly than conventional clinical end points; they hold the potential to substantially accelerate product development in certain disease. This review is mainly focused on the role biomarkers in cancer drug development process and the literature was collected from articles published in PUBMED, Medline, and the Cochrane Database of Systemic Reviews.
\end{abstract}

Keywords: Biomarkers; Clinical trials; Drug Development; Cancer.

\section{Introduction}

Biomarker is a substance/structure/process that can be measured in the body or its products and influence or predict the incidence of outcome or disease. Apart from the fact that biomarkers are very useful tool in accessing the progression of the disease and determine the treatment of success, there has being a trend in ongoing interest of role of biomarkers in preparation and testing the safety and efficacy of anticancer drug. Chemotherapy development has started since 1940s, where antifolate and nitrogen mustard were used for first time [1] since then cancer drug development has transformed and now, we have drugs which targets the specific molecular targets (receptors) which are as a result of mutation that caused cancer. The slow progression of anticancer drug development and high rates of failure is believed to be lack of predictive animal models which reflects the human experience. [2] According to a systematic review the use of biomarkers in phase I trials has increased over the period from 1991 to 2002 [2] and study has also shown that the biomarkers are used to limited extent for dose selection in phase II clinical trials.

\section{Biomarkers in clinical trails}

Clinical trials conducted among the patients with Gastrointestinal Stromal Tumors (GIST), a rare form of soft tumor to evaluate role of Imatinib in Patient survival rate and safety and efficacy of drug has shown promising results. [3] In another research the role of lapatinib was accessed in patients suffering from heavily treated advanced malignancies showing overexpression of tumor markers ErB1 and /ErB2 and results showed lapatinib exhibited preliminary of biological and clinical activities. A study shows synergistic effect of ZH1 when combined with gemcitabine, both combined has strong anticancer activity as they both enhances the apoptotic pathway in the body which is often disturbed in cancer.[4] Potential antitumor activities of glaucocalyxin A (GLA), an ent-kaurene diterpenoid isolated from Rabdosia japonica were accessed using gas chromatography-mass spectrometry(GC/MS) and liquid chromatography-mass spectrometry(LC/MS) based metabolic profiling and has shown positive results.[5] Lower class of small molecular therapeutics like histone deacetylase inhibitors (HDACi) are now approved by food and drug administration (FDA) as an anticancer agent. These drugs act as transcriptional repressors by removing acetyl group from histones. Hence combination of HDACi and anticancer drugs has shown promising results.[6] 
Proteomic technologies are extensively used in anticancer drug discovery and development.[7] Anthoporaanjunae is an antitumor peptide obtained from sea anemone, in a recent study this antipeptide has being shown to cause an inhibitory effect on prostate cancer DU-145 cells proliferation using methylthiazolyldiphenyl-tetrazolium bromide assay. This peptide increases the expression of apoptotic caspases and inhibits BCL-2 which is an antiapoptotic agent already found in our body and frequently over expressed in cancer conditions. [8] Advances in fields of inorganic chemistry have opened the pathways to new and effective anticancer regimen. Certain synthetic complexes have anticancer activity shown in a recent study. Where complex iridium upon irradiation with white light shows highly cytotoxic against BEL 7402 cells. The mechanism being it inhibits cell growth by reactive oxygen species (ROS) mediated mitochondrial destruction and targeting tubular pathways. The researchers are in progress and it is believed that this complex will be used to develop multi-cancer drugs. [9]

Phase III trials with ipilimumab showed an improved survival in patients with metastatic melanoma. [10] Numerous clinical trials are having taken place in past few years and results are positive in most cases. Moreover, the advanced radiologic imaging techniques are now extensively used and are accurate to detect the cancer at very early stage and leads to low cancer related deaths.[11] however researches are still going on. As deduced from above few researches mentioned it is understood that tumor markers play an inevitable role in diagnosis, treatment, prediction of treatment outcome. Some of the identified biomarkers played an important role in drug development are described in Table -1 .

\section{Biomarkers in cancer}

The use of biomarkers in clinical research has paved way for the discovery of the progression and in turn, the treatment of various diseases. For example, oncology is one field wherein the role of biomarkers has created a major impact. Despite the recent decline in the incidence of cancer, longterm mortality rates remain unchanged. One of the most important factors in the survival of cancer is detection at an early stage. Clinical assays that detect the early events of cancer offer an opportunity to intervene and prevent cancer progression. Biomarkers are important molecular signatures of the phenotype of a cell that aid in early cancer detection and risk assessment. [12] Although tumor markers greatly improve diagnosis, the invasive, unpleasant, and inconvenient nature of current diagnostic procedures limits their application. [13] Hence, there is a great need for the identification of non-invasive biomarkers for the early detection of tumors. Recent studies have found that that serum and plasma contain many stable miRNAs derived from various tissues/organs, and that the expression profile of these miRNAs shows great promise as a novel non-invasive biomarker for diagnosis of cancer and other diseases. [14] Another study showed the use of biomarkers in distinguishing between colon and ovarian cancer. These types of cancer maybe often difficult to differentiate in ovarian masses, in peritoneal carcinomatosis, and in metastases to distant lymph nodes. Misdiagnosis in this context may result in delayed identification of the primary lesion or misdirected clinical procedures. It may also lead to inappropriate therapy because metastatic colon cancer is generally treated with 5-FU, whereas ovarian cancer is most often treated with paclitaxel and a platinum agent $[15,16]$. This study indicates that villin and perhaps moesin should be strongly considered for clinical use in distinguishing colon and ovarian carcinomas. [17]

Recent advances in biomarkers research had provided a reliable, non-invasive method for the early detection of cancer, and thereby to improve the chances for successful therapy and preclude the use of an invasive biopsy. While screening the prostate cancer, Prostate Specific antigen (PSA) is one of the most important biomarkers but it leads to over-diagnosis and over-treatment because of the less specificity while screening. According to Niels Asger Jakobsen et al. there are lot of novel biomarkers came into the picture for screening the prostate cancer but still they are not put forward to clinical practices instead they are using PSA as a biomarker. So, to minimize this bias usage of the novel biomarkers like four-kallikrein panel, fPSA and PCA3 into the Prostate Cancer Prevention Trial Prostate Cancer Risk Calculator (PCPTrc) and the European Randomized Study of Screening for Prostate Cancer (ERSPC) multivariable prediction model can help clinicians to diagnose and treat precisely. [18] In 2011 Pritchard et al. exclaimed that circulating, cell-free micro RNAs (miRNAs) is 
one of the important biomarkers for the screening of cancers particularly leukemias, lymphomas and tumors which shows secondary effect on blood cells. [19] But some studies prove that there are different types of miRNAs which can be used as biomarkers for various cancer types including detection of breast cancer in the early stages. Detection of breast cancer in early stages is very important which can be attained by the level of serum miRNAs. [19] Lack of efficacy and safety of a drug which is developed and manufactured without using any clinical biomarker is the major drawback in clinical trials therefore FDA has launched the Critical PathInitiative to develop drugs using biomarkers for proper diagnosis and treatment in cancer therapy. Biomarkers can be used in different phases of drug discovery and development they are Target identification and validation, Lead identification and optimization, Preclinical development, Early-stage clinical development and Late-stage clinical development. There are different kinds of biomarkers namely

1. Target engagement: a drug which is been bound by fraction of target binding sites

2. Pharmacodynamics: Drug effects on the human body to assess whether the downstream pathway or biological process regulated by a drug target is perturbed upon drug administration.

3. Disease activity: Effects of a drug on a disease, which occur late in the pathophysiologic cascade and are linked to clinical benefit.

4. Surrogate endpoint: Substitution for a clinical endpoint and prediction of clinical benefit with certainty.

Safety biomarkers are one of the important biomarkers, but they are still in phase III clinical trials. [21] Mutations that occur in some of the biochemical substances present in human body such as BRAF V600E, anaplastic lymphoma kinase (AKL), and BCR-ABL translocation product may act as clinical biomarkers in melanoma, lung cancer and chronic myelogenous leukemia (CML) respectively, these biomarkers are selected for molecular anticancer therapy. Some of the identified biomarkers and their related to anticancer along with its mechanism of action (MOA) of drugs that are provided in the table -2 . To avoid usage of non-reactive and toxic drugs for cancer therapy it's better to know about the relative biomarker to find key molecular pathways for targeting. Recently, some of the antibodies are used for the treatment of cancer by targeting Fc region to trigger immune response with the help of T-cell activation. For example, ertumaxomab is a rat-murine hybrid monoclonal antibody tested and confirmed in the phase I and phase II clinical trials that it is used for breast cancer therapy in those patients showing HER2 positive. [22]

There is certain type of drugs which lead to tissue damage and increase the level of novel urinary biomarkers in the body. For example, vancomycin is an antibiotic used for the treatment of acute kidney injury (AKI), but toxicity of this drug due to high dose and increased treatment duration may lead to histopathological damage and release of novel urinary biomarkers these studies are done on rat. [22] From above, it is clear, that certain tumor biomarkers are making a major contribution to cancer detection and patient management. Indeed, in some situations, biomarkers can be used as the only available criterion for clinical decision making.

In the future, the trend will be to simultaneously measure multiple biomarkers with technologies such as microarray, multiplex PCR, multigene sequencing and eventually whole genome analysis. The use of multiple markers can potentially capture more fully, intra-tumor heterogeneity and provide more comprehensive clinical information and which helps to produce new anti-cancer drugs in to the market. 
DOI: 10.21522/TIJBMS.2016.03.02.Art001

ISSN: $2519-500 \mathrm{X}$

Table 1. Biomarker/Surrogate endpoints that have aided drug development

\begin{tabular}{|c|c|c|}
\hline Biomarker/Surrogate Endpoint & Type of Drug & Clinical Endpoint \\
\hline Blood pressure & Antihypertensives & $\begin{array}{l}\text { Stroke, atherosclerosis, heart } \\
\text { failure }\end{array}$ \\
\hline Cholesterol & LDL-lowering statins & $\begin{array}{l}\text { Coronary artery disease, heart } \\
\text { attacks }\end{array}$ \\
\hline Viral RNA & Antiretroviral agents & Survival, decrease in infections \\
\hline HbA1C, glucose & Antidiabetic agents & Diabetic neuropathy \\
\hline CD4+ T cells & $\begin{array}{l}\text { Antiretroviral agents, } \\
\text { Cytokines }\end{array}$ & $\begin{array}{l}\text { Sustained reduction in viral } \\
\text { RNA }\end{array}$ \\
\hline Intraocular pressure & Antiglaucoma agents & $\begin{array}{l}\text { Preservation of peripheral } \\
\text { vision }\end{array}$ \\
\hline Bone mineral density (BMD) & $\begin{array}{l}\text { Antiosteoporotic } \\
\text { agents }\end{array}$ & Fracture rate \\
\hline MRI scans & $\begin{array}{l}\text { Agents for treatment } \\
\text { of MS }\end{array}$ & $\begin{array}{l}\text { Decrease in rate of progression } \\
\text { disease }\end{array}$ \\
\hline CT scans for tumor size & Anticancer agents & Survival \\
\hline
\end{tabular}

Table 2. Table showing the biomarker identified in different types of cancers and drugs that has been used along with its action

\begin{tabular}{|l|l|l|l|}
\hline Cancer Type & $\begin{array}{l}\text { Biomarker } \\
\text { identified }\end{array}$ & Related Drug Used & Drug Action \\
\hline Lung cancer & ALK & Ceritinib & $\begin{array}{l}\text { Tyrosine kinase inhibitor of } \\
\text { ALK }\end{array}$ \\
\hline Colorectal cancer & EGFR & Panitumumab & $\begin{array}{l}\text { Humanized monoclonal } \\
\text { antibody directed against EGFR }\end{array}$ \\
\hline Breast cancer & HER2 & Lapatinib & $\begin{array}{l}\text { Reversible tyrosine kinase } \\
\text { inhibitor of EGFR, HER2 }\end{array}$ \\
\hline
\end{tabular}

\section{References}

[1]. Chabner BA, Roberts TG Jr. Timeline: Chemotherapy and the war on cancer. Nature reviews 2005, 5(1):65-72.

[2]. Bernardo HL, Goulart, Clark JW, Pien HH, et al. Trends in the Use and Role of Biomarkers in Phase I Oncology Trials. Clin Cancer Res. 2007 Nov 15; 13(22 Pt 1):6719-26.

[3]. Yeh CN, Chen TW, Wu TJ, et al. Treatment of patients with advanced gastrointestinal stromal tumor of small bowel: implications of imatinib mesylate. World Journal of Gastroenterology. 2006; 12(23):3760-5.

[4]. Guo J, Li Y, Lam CWK. ZH-1 enhances the anticancer activity of gemcitabine via deoxyribonucleotide synthesis and apoptotic pathway against A549 cells. Food Chem Toxicol. 2018; 15(18) 30221-7.

[5]. Liu Y, Lu S, Zhao L, Dong X. Effects of glaucocalyxin an on human liver cancer cells as revealed by GC/MS- and LC/MS-based metabolic profiling. Anal Bioanal Chem. 2018; 410(14): 3325-3335.

[6]. Suraweera A, O'Byrne KJ, Richard DJ. Combination Therapy with Histone Deacetylase Inhibitors (HDACi) for the Treatment of Cancer: Achieving the Full Therapeutic Potential of HDACi. Front Oncol. 2018 Mar 29; 8:92.

[7]. Jain KK. Proteomics-based anticancer drug discovery and development. Technol Cancer Res Treat. 2002 Aug; 1(4):231-6.

[8]. Wu ZZ, Ding GF, Huang FF, et al. Anticancer Activity of Anthopleuraanjunae Oligopeptides in Prostate Cancer DU-145 Cells. Mar Drugs. 2018 Apr 12; 16(4): 125.

[9]. Zhang WY, Yi QY, Wang YJ, et al. Photo induced anticancer activity studies of iridium (III) complexes targeting mitochondria and tubules. Eur J Med Chem. 2018 Apr 7; 151:568-584.

[10]. Jochems A, Leeneman B, Franken MG, et al. Real-world use, safety, and survival of ipilimumab in metastatic cutaneous melanoma in The Netherlands. Anticancer Drugs 2018; 29(6):572-578. 
[11]. Loong MT. Emerging Trends in Oncology: Greater Role for Radiology. Journal of Medical Diagnostic Methods.2012; 10(4172):2168-9784.

[12]. Robert S. Negm, Mukesh Verma, Sudhir Srivastava. The promise of biomarkers in cancer screening and detection. TRENDS in Molecular Medicine 2002; 8 (6); 221- 256.

[13]. Duffy MJ. Role of tumor markers in patients with solid cancers: a critical review. Eur J Intern Med 2007; $18: 175-184$.

[14]. Xi Chen, Yi Ba, Chen-Yu Zhang "Characterization of microRNAs in serum: a novel class of biomarkers for diagnosis of cancer and other diseases" Cell Res. 2008 Oct; 18(10):997-1006.

[15]. Moertel C. G. Chemotherapy for colorectal cancer. N. Engl. J. Med., 1994; 330:1136-1142.

[16]. Qazi F., McGuire W. P. The treatment of epithelial ovarian cancer. CA Cancer J. Clin., 1995; 45:88-101.

[17]. Satoshi Nishizuka, Sing-Tsung Chen, Fuad G. Gwadry, Jes Alexander, Sylvia M. Major. Diagnostic Markers That Distinguish Colon and Ovarian Adenocarcinomas: Identification by Genomic, Proteomic, and Tissue Array Profiling. Molecular Biology, Pathobiology, and Genetics, 2003; 63 (1): 5243-5250.

[18]. Jakobsen NA, Hamdy FC, Bryant RJ. Novel biomarkers for the detection of prostate cancer. J Clin Urol. 2016; 9(2 Suppl):3-10.

[19]. Pritchard CC, Kroh E, Wood B, et al. Blood cell origin of circulating microRNAs: a cautionary note for cancer biomarker studies. Cancer Prev Res (Phila). 2011;5(3):492-497.

[20]. Shimomura A, Shiino S, Kawauchi J, et al. Novel combination of serum microRNAs for detecting breast cancer in the early stage. Cancer Sci. 2016; 107(3):326-34.

[21]. Xuemei Zhao, Vijay Modur, Leonidas N. Carayannopoulos, Omar F. Laterza Clinical Chemistry Nov 2015, 61 (11) 1343-1353;

[22]. Smith AD, Roda D, Yap TA. Strategies for modern biomarker and drug development in oncology. $J$ Hematol Oncol. 2014; 7:70. Published 2014 Oct 3. doi:10.1186/s13045-014-0070-8. 